\title{
GAP-43 in Growth Cones Is Associated with Areas of Membrane That Are Tightly Bound to Substrate and Is a Component of a Membrane Skeleton Subcellular Fraction
}

\author{
Karina F. Meiri ${ }^{1}$ and Phillip R. Gordon-Weeks ${ }^{2}$ \\ 'Departments of Pharmacology and Anatomy and Cell Biology, SUNY Health Science Center, Syracuse, New York 13210 , \\ and 'Department of Anatomy and Human Biology, King's College London, London WC2R 2LS, United Kingdom
}

\begin{abstract}
To ascertain the subcellular localization of the growth-associated protein GAP-43 in growth cones, we isolated growth cones from the forebrains of neonatal rats. Anti-GAP-43 immunoreactivity in these isolated growth cones (IGCs) resembled that seen in cultured growth cones in 2 respects: First, in substrate-attached IGCs, as in cultured growth cones, immunoreactivity was intracellular, punctate, and extended throughout the IGCs and their processes. Second, when IGCs were dislodged from this substrate, patches of membrane that were highly immunoreactive for GAP-43 were left behind. Extracting IGCs in nonionic detergents revealed that almost all the particulate GAP-43 colocalized with a membrane skeleton fraction: It was not present in the cytoskeleton. The association of GAP-43 with the membrane skeleton was not due to nonspecific aggregation, nor was it calcium dependent. Examination of this fraction under the electron microscope showed it to consist of membrane fragments associated with amorphous material that could be visualized with tannic acid. Immunoelectron microscopy showed that anti-GAP-43 immunoreactivity was localized to this amorphous material. Fodrin, talin, and a-actinin immunoreactivity could be detected in the membrane skeleton fraction, and actin and tubulin were also identifiable from SDS gels. The association of GAP-43 with the membrane skeleton, which by analogy with other cell types is involved in the dynamic regulation of cell shape, implies that GAP-43 in growth cones may be involved in this function.
\end{abstract}

The growth-associated protein GAP-43 has both its synthesis and axonal transport highly induced in neurons that are extending axons during the development of the nervous system and in peripheral neurons that are regenerating following injury (Skene and Willard, $1981 \mathrm{a}$; Kalil and Skene, 1986). In contrast, GAP-43 is not induced in the damaged axons of neurons that do not normally regenerate, such as those of the mammalian CNS (Skene and Willard, 1981a), suggesting that it may be performing a function involved in axon outgrowth (Willard et al., 1987).

\footnotetext{
Received Mar. 20, 1989; revised June 15, 1989; accepted July 25, 1989.

This work was supported by grants from NATO (K.F.M. and P.G.W.), the National Institutes of Health NS 26091 (K.F.M.), and the Wellcome Trust (P.G.W.). We thank Drs. R. Bloch, J. Krekorian, and R. Cheney for their gifts of antibodies; Dr. J. Taylor and D. Burdick for experimental contributions; L. Bickerstaff for assistance, K. Bugg and K. Szumowski for help with electron microscopy; Dr. J. Schwob for careful reading of the manuscript and helpful comments; and P. Pisarek for secretarial help.

Correspondence should be addressed to Dr. Karina F. Meiri, Department of Pharmacology, SUNY Health Science Center at Syracuse, Syracuse, NY 13210. Copyright (c) 1990 Society for Neuroscience $0270-6474 / 90 / 010256-11 \$ 02.00 / 0$
}

The induction of GAP-43 in developing and regenerating neurons probably reflects its concentration in the growth cone that is elaborated at the leading edge of growing axons (Meiri et al., 1986, 1988; Skene et al., 1986). Since the growth cone is uniquely specialized to perform the pathfinding and motility functions critical to successful axon outgrowth (Bray, 1982; Letourneau, 1982), the observation in tissue culture that anti-GAP-43 immunoreactivity increases with the rate of translocation of growth cones across a substrate (Johnson et al., 1987) lends further support to the hypothesis that GAP-43 is performing a critical outgrowth-related function in that structure.

The goal of this study was to determine the subcellular localization of GAP-43 in growth cones so that we can begin to investigate its interactions with other growth cone proteins. Although previous biochemical studies had described GAP-43 as an integral membrane protein based on its behavior in detergents and salts (Skene and Willard, 1981a, b; Dosemeci and Rodnight, 1987), the primary sequence of GAP-43 contains no membrane-spanning regions (Basi et al., 1987; Karns et al., 1987). Furthermore, these experiments utilized subcellular fractions that probably contained other structures such as cytoskeletons as well as membranes, so that questions still remain as to the exact intracellular location of GAP-43 in the growth cone and its interactions with other proteins. Immunochemical localization at the light microscope level shows that in growth cones of cultured neurites GAP-43 immunoreactivity is punctate (Meiri et al., 1986, 1988). Some of this appearance is undoubtedly due to association of GAP-43 with vesicles; under the electron microscope immunoreactivity of the GAP-43 homolog B-50 was seen on vesicles in presynaptic terminals (Gispen et al., 1985). Immunoelectron microscopy of GAP-43 in growth cones isolated from neonatal rat brain showed immunogold labeling in areas adjacent to the plasma membrane (van Lookeren Campagne et al., 1989). However, the resolution was not sufficient to precisely localize GAP-43, and moreover, immunolocalization experiments such as these do not provide information about the other proteins with which GAP-43 colocalizes.

Subcellular fractions enriched in growth cones that have been prepared from forebrains of fetal (Pfenninger et al., 1983) or neonatal rat brains (Gordon-Weeks and Lockerbie, 1984) may be used as a model system in which to study GAP-43 distribution and interactions. The "isolated" growth cone preparation that we have used (Gordon-Weeks and Lockerbie, 1984; Gordon-Weeks, 1987a) has 2 features to recommend it for these studies: First, the morphology within the isolated growth cones (IGCs) is well preserved, demonstrating that protein/protein interactions are not significantly disrupted during the fraction- 
ation process (Gordon-Weeks and Lockerbie, 1984). Second, like growth cones in tissue culture (Hume et al., 1983; Young and Poo, 1983), these IGCs are able to take up gamma-aminobutyric acid and noradrenaline and subsequently to release them following depolarization with potassium, implying that the preparation retains a degree of physiological integrity (Gordon-Weeks et al., 1984; Lockerbie et al., 1985).

In this study we have: (1) characterized anti-GAP-43 immunoreactivity in isolated growth cones attached to a substrate and compared it with the distribution seen in regenerating neurites in tissue culture, (2) demonstrated the localization and enrichment of GAP-43 in a detergent-insoluble membrane skeleton prepared from isolated growth cones, (3) described the ultrastructure of this fraction under the electron microscope and investigated the localization of GAP- 43 by immunoelectron microscopy, (4) determined the protein composition of this membrane skeleton fraction and used antibodies to identify specific constituents, and (5) examined whether GAP-43 association with the membrane skeleton is calcium dependent. The results are discussed with reference to possible functions of GAP-43 in the motile growth cone. A preliminary report of some of this work has appeared elsewhere (Meiri and Gordon-Weeks, 1987).

\section{Materials and Methods}

Preparation of subcellular fractions. IGCs were prepared from postnatal day $\mathrm{I}-4(\mathrm{Pl}-4$; day of birth is $\mathrm{P} 1)$ rat forebrains as described previously (Gordon-Weeks, 1987a, b), with the following modifications: Material banding at the interface between the sample and the 7\% Ficoll solution following the first density gradient step was diluted 1:5 by adding Krebs' solution dropwise while stirring over ice. [Krebs' solution contained (in mm): $145 \mathrm{NaCl}, 5 \mathrm{KCl}, 1.3 \mathrm{MgCl}_{2}, 1.2 \mathrm{CaCl}_{2}, 1.2 \mathrm{NaH}_{2} \mathrm{PO}_{4}, 10$ glucose, 20 HEPES, pH 7.4).] The resultant suspension was centrifuged at 14,500 $\times g_{\mathrm{av}}$ for $50 \mathrm{~min}$ at $4^{\circ} \mathrm{C}$, and the pellet resuspended in Krebs' solution at a concentration of about $3 \mathrm{mg} / \mathrm{ml}$. This fraction is designated "IGCs." In some experiments $1.5 \mathrm{~mm}$ EGTA was added to all the solutions used to prepare the isolated growth cones. In these experiments also, calcium was ormitted from the Krebs' solution and $1.5 \mathrm{~mm}$ EGTA substituted. "Particulate fractions" were prepared from IGCs by suspending them in a solution containing $10 \mathrm{~mm}$ Tris, $\mathrm{pH} 7.6,0.2 \mathrm{~mm}$ DTT, $1 \mathrm{mM}$ PMSF, $1 \mu \mathrm{g} / \mathrm{ml}$ leupeptin, and $1 \mu \mathrm{g} / \mathrm{ml}$ pepstatin (buffer A) and then centrifuging the suspension at $100,000 \times g_{a v}$ for $75 \mathrm{~min}$ at $4^{\circ} \mathrm{C}$. The pellet is designated the particulate fraction.

Cytoskeletons were prepared from particulate fractions of IGCs by resuspending the pellet in a buffer containing $24 \mathrm{~mm}$ HEPES pH 6.8, 2 $\mathrm{mM} \mathrm{MgCl}_{2}, 10 \mathrm{~mm}$ EGTA, $1 \mathrm{~mm}$ PMSF, and $2.5 \%$ Triton X-100 at a detergent: protein ratio of $166: 1$ (wt:wt), centrifuging the suspension and collecting the detergent insoluble fraction as described previously (Gordon-Weeks, 1987b). Detergent-insoluble membrane skeletons were prepared from particulate fractions of IGCs by resuspending them in buffer A containing 5\% Nonidet P-40 at a detergent : protein ratio of $5: 1$ (wt:wt). The detergent-insoluble proteins were then further fractionated by discontinuous sucrose density gradient centrifugation, as described previously (Moss, 1983) In using this method, the membrane skeleton is defined as proteins that are localized at the interface between the $10 \%$ and $30 \%$ sucrose layers.

The apparent molecular weight of membrane skeleton and associated GAP-43 was estimated from their sedimentation coefficients by continuous sucrose density gradient centrifugation using the procedure of Martin and Ames (1961), as follows: cither $30 \mu \mathrm{g} \mathrm{membranc} \mathrm{skclcton}$ proteins at a concentration of $2 \mu \mathrm{g} / \mu \mathrm{l}$ in buffer A, pure GAP-43 $(0.5 \mu \mathrm{g} /$ $\mu \mathrm{l}$ in buffer $\mathrm{A}), 10 \mu \mathrm{g}$ bovine serum albumin, or $10 \mu \mathrm{g}$ chymotrypsinogen was suspended in $0.05 \mathrm{M}$ Tris $\mathrm{pH} 7.5$ and then centrifuged on a linear 5-20\% sucrose gradient for $18 \mathrm{hr}$. In some experiments pure GAP-43 was added to the membrane skeleton fraction prior to centrifugation. The gradients (total volume $4.6 \mathrm{ml}$ ) were collected as 1-drop $(120 \mu \mathrm{l})$ aliquots which were immobilized on nitrocellulose paper by dot-blotting. The nitrocellulose was then either stained with naphthol blue-black to reveal the position of protein on the gradient or incubated with antiGAP-43 antibody to reveal the position of GAP-43. Immunoreactivity was visualized with biotinylated secondary antibody and a complex of avidin and biotinylated-horseradish peroxidase (Vectastain, Vector Laboratories).

Immunohistochemistry and electron microscopy. IGCs that had been allowed to settle onto polyornithine-coated coverslips (Gordon-Weeks and Lockerbie, 1984) were fixed with $2 \%$ formaldehyde, made permeable with $0.01 \%$ digitonin, and incubated with affinity-purified antiGAP-43 as before (Meiri et al., 1988). Anti-GAP-43 immunoreactivity was visualized with fluoresceinated goat anti-rabbit IgG (Cappell) and compared with that seen in the growth cones of neurites of embryonic superior cervical ganglia that had been treated similarly (Meiri et al., 1988). In 1 set of experiments, isolated growth cones, or in another set, the growth cones of cultured neurites, were dislodged from the substrate with a gentle stream of Krebs' buffer or culture medium, respectively, leaving behind patches of membrane that were strongly attached to the substrate. These membrane patches that remained attached to the coverslips were then fixed and prepared for immunohistochemistry, as described above.

For electron microscopy, membrane skeleton fractions that had been prepared from isolated growth cones by extractions in NP-40 followed by discontinuous sucrose gradient centrifugation, as described above, were fixed for $20 \mathrm{~min}$ in $3 \%$ glutaraldehyde in $0.12 \mathrm{M}$ sodium cacodylate

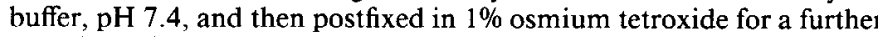
$15 \mathrm{~min}$ before staining with a saturated aqueous solution of uranyl acetate, dehydrating, and embedding in Epon. In some cases $0.2 \%$ tannic acid (Mallinckrodt) was added to the primary fixativc. For immunoclectron microscopy, membrane skeletons were incubated prior to fixation for $24 \mathrm{hr}$ at $4^{\circ} \mathrm{C}$ in affinity-purified anti-GAP-43 in PBS, pH 7.4, containing $5 \% \mathrm{BSA}$ and $0.01 \%$ digitonin, and then for the same time in 15 $\mathrm{nm}$ gold-conjugated anti-rabbit $\mathrm{IgG}$ secondary antibody (Janssen) diluted 1:5 in PBS-5\% BSA. Membrane skeletons were then fixed, stained, and sectioned as above.

A detailed account of the preparation of anti-GAP-43 antiserum, its affinity purification, and its characterization has appeared elsewhere (Meiri et al., 1986).

Gel electrophoresis and immunoblotting. Electrophoresis on 1-dimensional $10 \%$ linear or $3.5-15 \%$ gradient polyacrylamide SDS gels was according to the method of Laemmli (1970). Electrophoresis in 2 dimensions was performed as described previously (Meiri et al., 1986). Protcins that had been separated by SDS-PAGE were visualized by staining the gels either with Coomassie blue or with silver (Oakley et al., 1980). Scanning absorbance densitometry of silver-stained gels at $415 \mathrm{~nm}$ or Coomassie-stained gels at $578 \mathrm{~nm}$ utilized a Shimadzu densitometer. For densitometry, protein was loaded onto the gels so that the concentration was within the linear range of the response of the instrument. This was determined by loading different concentrations of molecular weight markers on gels, staining the gels, and then scanning them.

One-dimensional SDS gels were Western-blotted onto nitrocellulose paper for $1 \mathrm{hr}$ at $100 \mathrm{~V}$ using a BioRad minigel transfer apparatus. The nitrocellulose paper was then incubated with the following primary antibodies: affinity-purified anti-GAP-43; anti-fodrin, a generous gift of Dr. R. Cheney; anti-vinculin and anti-talin, generous gifts of Drs. J. Krekorian and R. Bloch; and anti-a-actinin (Sigma Chemical Co., St. Louis, MO). Immunoreactivity was visualized on Western blots with biotinylated secondary antibodies followed by biotin-avidin horseradish peroxidase. To perform scanning absorbance densitometry (at $415 \mathrm{~nm}$ ) on Western blots, the nitrocellulose paper was first soaked in cedarwood oil to make it transparent. The amount of GAP-43 loaded onto blots was adjusted so that the signal was not saturated and within the linear range of the densitometer. Protein concentrations were determined using a Coomassie-based assay (Bradford, 1976) with modifications (Read and Northcote, 1981; Gogstad and Krutnes, 1982) to enable measurement of membrane proteins. BSA was used as standard.

\section{Results}

\section{Localization of GAP-43 in IGCs}

\section{GAP-43 immunoreactivity in IGCS is intracellular and} punctate

GAP-43 immunoreactivity in the growth cones of neurites regenerating in tissue culture extends throughout the body of the growth cone and its filopodia (Meiri et al., 1988). When IGCs prepared from neonatal (P1-4) rat forebrains are allowed to settle onto a polyornithine-coated coverslip, they flatten out, become 
Figure 1. GAP-43 immunoreactivity in IGCs is intracellular and punctate. $a$, $b$, High-power fluorescent photomicrographs showing anti-GAP-43 immunoreactivity in IGCs that have attached to a polyornithine-coated coverslip. Immunoreactivity extends throughout the IGCs including the fine processes (arrows) and appears punctate (open arrows). Scale bars, $1 \mu \mathrm{m}$. $c$, IGCs that have been incubated with nonimmune IgG. $d$, Anti-GAP-43 immunoreactivity in a cultured superior cervical ganglion growth cone that has detached from its neurite. Punctate fluorescence is extensive throughout the growth cone, lamellipodia (arrows), and filopodia (arrowheads). Scale bar, $2 \mu \mathrm{m} . e$, Phase contrast photomicrograph of IGCs that have attached to polyornithine-coated coverslip. Single particles were counted, but aggregates (open arrows) were ignored. Arrows indicate particles that did not bind anti-GAP-43. $f$. Corresponding fluorescent photomicrograph demonstrating GAP-43 immunoreactive particles and particles that did not bind antibody (arrows). Scale bars, 20 $\mu \mathrm{m}$.
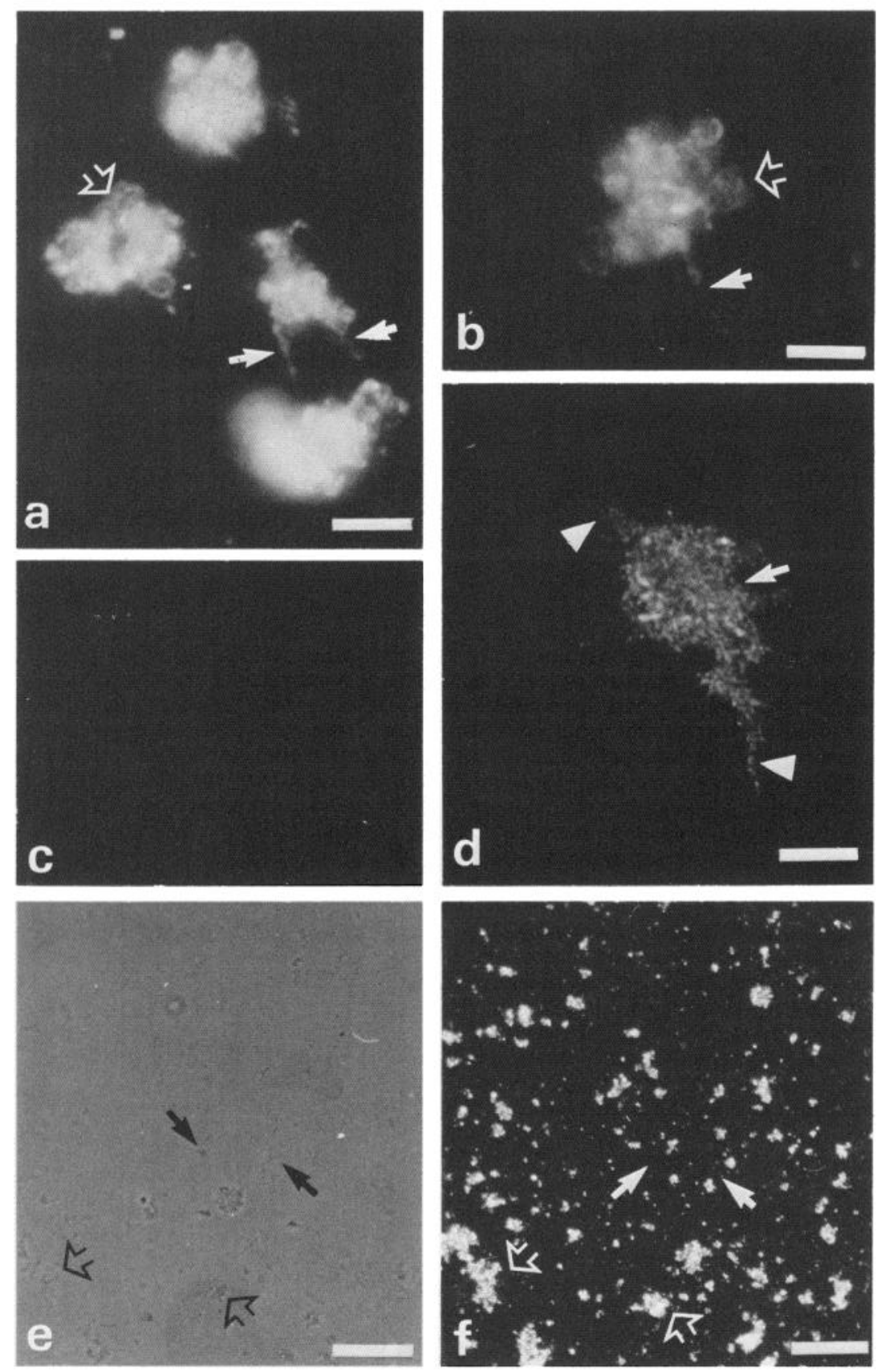

phase bright, and display fine filopodia-like processes (GordonWeeks and Lockerbie, 1984). When IGCs that had attached to a polyornithine substrate were fixed with $2 \%$ formaldehyde, permeabilized with $0.01 \%$ digitonin, and then incubated with anti-GAP-43, antibody bound to those large particles that had attached to the substrate and could be visualized with fluoresceinated secondary antibody. Under high power, as in cultured growth cones, the immunofluorescence looked punctate and extended throughout the particles and their processes (Fig. 1, $a$, $b$ ). In contrast, substrate-attached IGCs that had been fixed, but not made permeable, prior to incubation with antibody fluoresced only faintly (results not shown), suggesting that the fixation technique partially disrupts the membrane, as described previously (Meiri et al., 1988). This faint fluorescence was not seen when the IGCs were incubated with nonimmune IgG (Fig. $1 c$ ). Thus, the epitopes recognized by the anti-GAP-43 antibody are on the inside of the IGCs. The pattern of immunoreactivity seen in IGCs resembles that seen in growth cones in tissue culture: Figure $1 d$ shows anti-GAP-43 immunoreactivity in a growth cone that has detached from a superior cervical ganglion cell neuron that had been grown in tissue culture, demonstrating the characteristic punctate immunoreactivity that extends throughout the growth cone and filopodia.

To quantitate the number of substrate-attached particles that were GAP-43 immunoreactive, we photographed 14 separate fields of attached particles under both phase contrast and fluorescence microscopy (Fig. 1, e, f). We then aligned the photographs and marked single attached particles by impaling them with a pin. Aggregates of particles were not counted. Those particles which were also fluorescent were then scored by identifying pinholes on the fluorescent photomicrographs. We counted 14 separate fields of 150 particles each and estimated that 

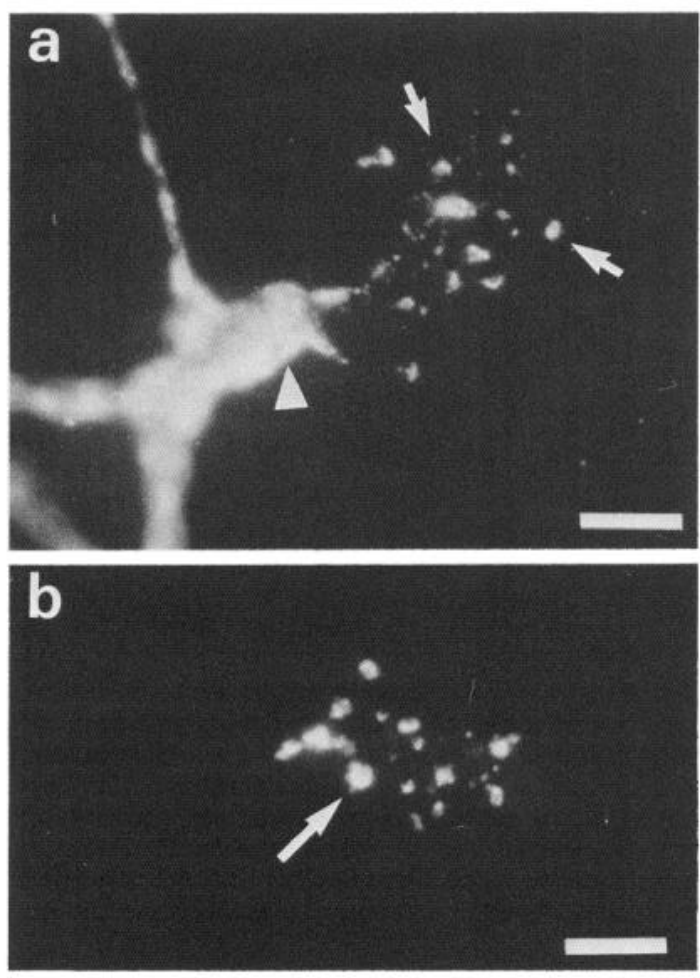

Figure 2. Anti-GAP-43 immunoreactivity is associated with patches of membrane that adhere tightly to substrate. Fluorescent photomicrographs: $a$, Anti-GAP-43 immunoreactivity in a cultured superior cervical ganglion neuron in which the growth cone has been dislodged from the substrate, leaving behind patches of membrane that are immunoreactive with anti-GAP-43 (arrows). The neurite remains intact, though out of focus (arrowhead). Scale bar, $2 \mu \mathrm{m}$. $b$, Anti-GAP-43 immunoreactivity in patches of membrane remaining when an IGC that had attached to a polyornithine-coated coverslip was dislodged with a stream of Krebs' buffer (see Methods). These patches were immunoreactive (arrow) even though the preparation was not permeabilized before incubation with antibody. Scale bar, $1 \mu \mathrm{m}$.

$74.41 \% \pm 6.01 \%( \pm 1 \mathrm{SD})$ of all attached particles were immunoreactive with anti-GAP- 43 and therefore were identifiably of neuronal origin.

\section{GAP-43 immunoreactivity is associated with areas of membrane that adhere tightly to substrate}

The growth cones of sympathetic ganglion neurites growing in tissue culture could be perturbed with a gentle stream of culture medium, so that most of the body of the growth cone was dislodged, with the exception of patches of membrane that remained tightly attached to the substrate. Anti-GAP-43 antibody bound to these substrate-associated areas of membrane even if they had not been treated with digitonin (Fig. $2 a$ ), indicating that the cytoplasmic face of the membrane-attached fragments was exposed to the medium after the growth cones had been dislodged. Similarly, IGCs that had attached to a polyornithine substrate and then been dislodged with a gentle stream of Krebs' buffer left behind patches of membrane that also bound antiGAP-43 antibody without permeabilization by digitonin (Fig. $2 b$ ). These results suggest that at least some of the punctate staining with anti-GAP-43 in growth cones is due to association of GAP- 43 with areas of growth cone membrane that are tightly attached to the substrate.

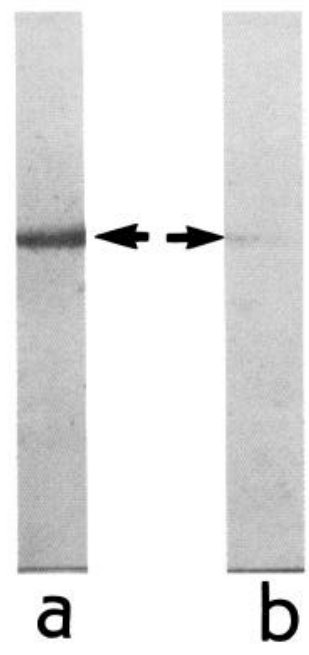

Figure 3. GAP-43 is not associated with the cytoskeleton. Western blots that have been incubated with $0.08 \mu \mathrm{g} / \mathrm{ml}$ affinity-purified antiGAP-43 antibody. Immunoreactivity was visualized with biotin-avidin HRP. $a$, Protein $(15 \mu \mathrm{g})$ from the particulate fraction of IGCs that is soluble in Triton X-100 at a detergent : protein ratio of $166: 1 . b$, Protein $(15 \mu \mathrm{g})$ from the Triton-insoluble cytoskeleton. GAP-43 immunoreactivity is indicated with arrows. Less than $10 \%$ of the total immunoreactivity is associated with the cytoskeleton fraction.

GAP-43 is associated with, and enriched in, a membrane skeleton fraction prepared from isolated growth cones

The IGCs were further fractionated in the hope of enriching for GAP-43. The strategy was to incubate IGCs with nonionic detergents under specific conditions and then to examine the detergent-insoluble fractions, since detergents will differentially affect membranes. For example, in myotubes, patches of membrane remain attached to the substrate after most of the membrane has been solubilised with detergent (Bloch, 1984). When the IGC fraction was centrifuged at $100,000 \times g_{\max }$ for $75 \mathrm{~min}$, it produced a pellet, which is designated the particulate fraction, and a supernatant. Approximately $58 \%$ of all growth cone GAP43 is pelleted by this step, the rest remaining in the supernatant (Meiri and Gordon-Weeks, 1987).

A cytoskeletal fraction was generated by extracting the particulate fraction with Triton X-100 at a detergent : protein ratio of 166:1 (wt:wt) (Gordon-Weeks, 1987b). The resultant pellet contains most of the tubulin and actin in IGCs. Comparing Western blots of proteins that had been solubilized by Triton X-100 (Fig. $3 b$ ) with proteins from the Triton-insoluble cytoskeleton (Fig. $3 a$ ) showed that very little (less than $10 \%$ as judged by densitometry of Western blots) of GAP-43 immunoreactivity was associated with the detergent-insoluble cytoskeleton. We next made a membrane skeleton by treating particulate fractions of IGCs with Nonidet P-40 (NP-40) at a detergent : protein ratio of 5:1 (wt:wt) and then separating the NP-40-insoluble proteins on a discontinuous sucrose gradient, as described previously by Moss (1983); the membrane skeleton fraction is localized at the interface between $10 \%$ and $30 \%$ sucrose layers. We demonstrated that GAP-43 is enriched in this membrane skeleton fraction in 2 ways. First, densitometric analysis of Coomassie-stained 2D gels showed that approximately 4 times more than GAP43 was present in this membrane skeleton than in the particulate fraction from IGCs (Fig. 4, $a, b$ ). Second, aliquots of the discontinuous sucrose gradient used to prepare the membrane skel- 
Figure 4. GAP-43 is enriched in the membrane skeleton of IGCs. $a$, Coomassie-stained, 2-dimensional IEF gel of $10 \mu \mathrm{g}$ of protein from IGC particulate fraction. The area under the peak due to GAP-43 staining (arrow) was estimated from scanning densitometry at $578 \mathrm{~nm}$ as 1208.2 arbitrary units. $b$, Coomassie-stained, 2-dimensional IEF $\mathrm{gel}$ of $10 \mu \mathrm{g}$ of protein from the IGC membrane skeleton. Similar densitometry estimated the area under the GAP43 peak as 5829.4 arbitrary units; an enrichment of 4.82-fold. In each case the acid end of the gel is oriented to the left. Standards indicated at the righthand side by dots are 180,116,97, 58, $48,48,36$, and $26 \mathrm{kDa}$.

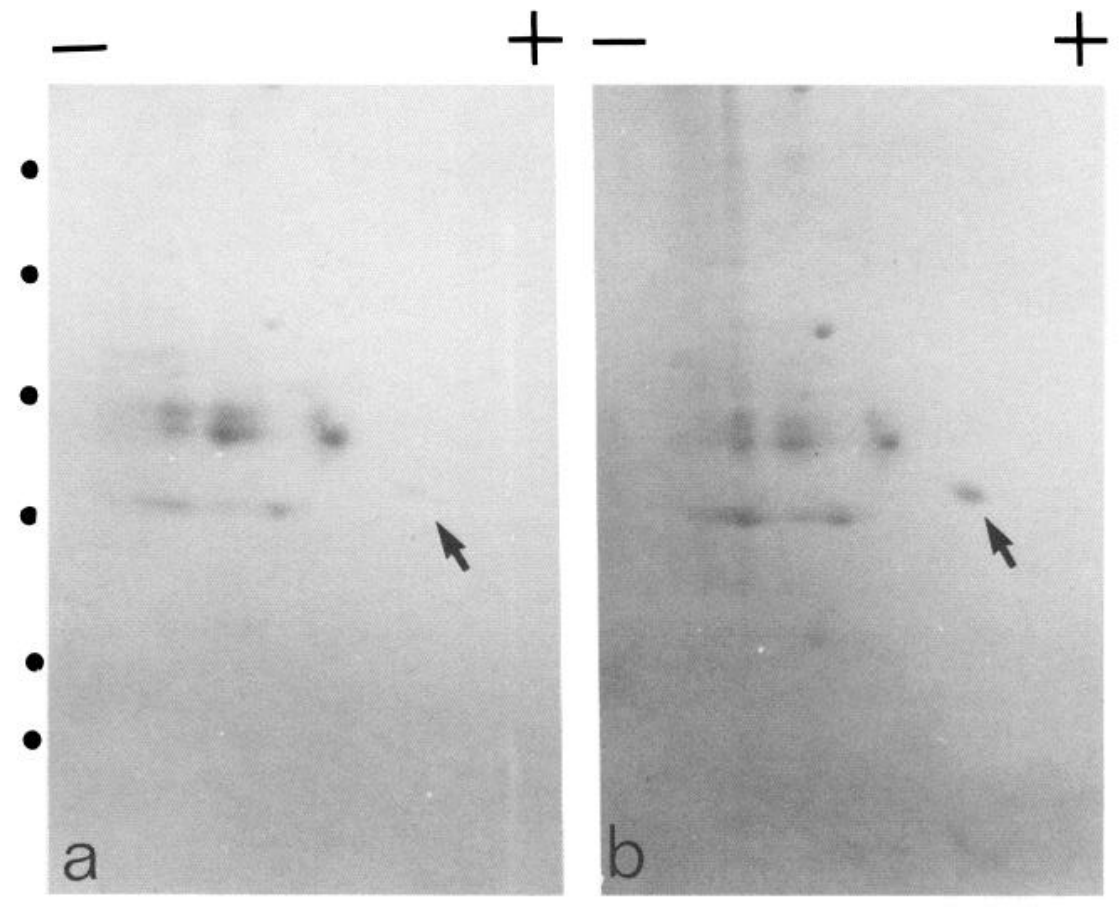

eton were analyzed on Western blots of $10 \%$ SDS gels for antiGAP-43 immunoreactivity. Almost all the NP-40-insoluble GAP-43 was localized in the membrane skeleton fraction (Fig. 5).

Comparison of proteins from the membrane skeleton (collected at the 10-30\% interface of the sucrose gradient) with proteins from the pellet at the bottom of the gradient showed that several proteins of molecular weights of approximately 480 (estimated), 290, 220, 105, 69, 50, 38, and $33 \mathrm{kDa}$ were also specifically localized or enriched in the membrane skeleton fraction (Fig. 6). Some proteins that were present in the pellet as well as the membrane skeleton were identifiable by their molecular weights, notably actin and tubulin (Fig. 6). (Although a Coomassie-stained gel is illustrated, silver staining of similar gels did not reveal extra proteins that were not stained with Coomassie blue.)

The association of GAP-43 with proteins of the membrane skeleton is specific

GAP-43 association with the membrane skeleton is not due to aggregation

To determine whether the apparent enrichment of GAP-43 in the membrane skeleton fraction was due to an artifactual, nonspecific entrapment caused by the experimental procedure, we performed continuous density gradient centrifugation analysis (Martin and Ames, 1961). To identify protein-containing fractions, aliquots of the gradients were dot-blotted onto nitrocellulose paper which was then stained with naphthol blue black (see Methods). To calibrate the gradients, chymotrypsinogen and BSA were centrifuged separately and migrated with Svedberg units of 2.7 and 4.5, respectively, corresponding to their monomeric molecular weights of 24 and $68 \mathrm{kDa}$ (Fig. $7 \mathrm{a}$ ). When pure GAP-43 was centrifuged alone, it migrated at $5.7 \mathrm{~S}$, equivalent to a molecular weight of $120 \mathrm{kDa}$ (Fig. $7 \mathrm{~b}$ ), which is different from its monomeric molecular weight of $24 \mathrm{kDa}$. A similar increase in the apparent molecular weight of GAP-43 has also been reported to occur during gel filtration (McIntosh et al., 1989); its cause is not yet known. The anomalous migration of GAP-43 on SDS gels has been attributed to aggregation (Benowitz et al., 1987), its large axial ratio (Masure et al., 1986), acidic pI, or low detergent binding capabilities (Jacobson et al., 1986; Benowitz et al., 1987). When membrane skeleton proteins were centrifuged on the gradient, all of the anti-GAP-43 immunoreactivity and all of the protein migrated at $20 \mathrm{~S}$, which corresponds to an apparent molecular weight of $>10^{6} \mathrm{kDa}$ (Fig. $7 \mathrm{c}$ ), indicating that the protein components of the subcellular fraction remained as a supramolecular aggregate during centrifugation. To determine whether this was because GAP-43 was itself aggregating under these conditions, we added pure GAP43 to the membrane skeleton prior to centrifugation. The amount of pure GAP-43 that was added was adjusted to be approximately equal to that present in the membrane skeleton, as judged by immunoreactivity. After centrifugation the analysis of dotblotted aliquots showed that GAP-43 immunoreactivity was present at $5.7 \mathrm{~S}$ as well as at $20 \mathrm{~S}$, where the membrane skeleton sediments. From densitometric analysis of these blots we estimated that $54 \%$ of the GAP-43 immunoreactivity was now detectable at $5.7 \mathrm{~S}$ (Fig. $7 d$ ). This result supports the interpretation that GAP-43 is not aggregating nonspecifically with the membrane skeleton.

\section{GAP-43 association with the membrane skeleton is not calcium dependent}

In some experiments we compared the association of GAP-43 with the membrane skeleton fraction in the presence and absence of calcium by modifying the preparation of IGCs and the subsequent isolation of membrane skeletons so that any calcium was omitted and $1.5 \mathrm{~mm}$ EGTA added to each solution. The amount of GAP-43 that partitioned into the pellet when a particulate fraction was prepared from IGCs was not affected by the presence of EGTA. Similarly, the presence of EGTA had 


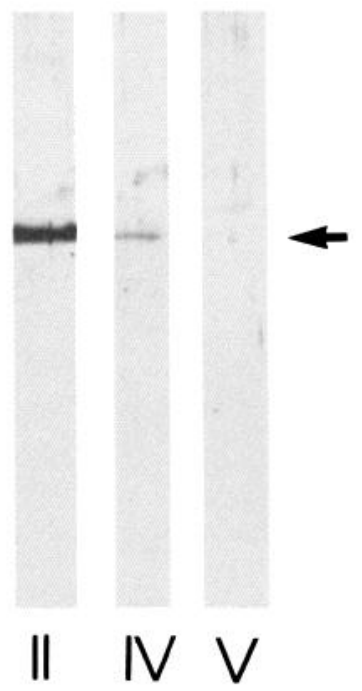

Figure 5. Particulate GAP-43 in growth cones is exclusively localized in the membrane skeleton fraction. Western blots of $10 \%$ SDS gels of fractions from the discontinuous sucrose gradients used to prepare membrane skeletons from IGCs. Anti-GAP-43 immunoreactivity (arrow) was visualized with biotin-avidin HRP. $I I$, The $10-30 \%$ interface (where the membrane skeleton is localized); $I V$, the $30-50 \%$ interface; $V$, protein that is pelleted through the gradient. In each case $15 \mu \mathrm{g}$ of protein was loaded.

no effect on the amount of GAP-43 fractionating with the membrane skeleton (Fig. 8).

\section{Ultrastructure and GAP-43 association with the membrane} skeleton fraction revealed by immunoelectron microscopy

Electron microscopy revealed that the membrane skeleton fraction from isolated growth cones was composed of membrane fragments and vesicles associated with clumps of amorphous fuzzy material that could be visualized with tannic acid staining (Fig. 9, $a$ and inset). No other identifiable organelles or filaments were present. The membrane-bound vesicles had an average diameter of $0.213 \mu \mathrm{m} \pm 0.077 \mu \mathrm{m}(x= \pm 1 \mathrm{SD}, n=50)$, which suggests that they are fragments of IGCs, since the latter have diameters of 2-5 $\mu \mathrm{m}$ (Fig. 1, $a, b$; see also Gordon-Weeks and Lockerbie, 1984). When anti-GAP-43 binding to the membrane skeleton fraction was visualized under the electron microscope with gold-conjugated secondary antibody, immunoreactivity was apparent in clusters that were associated with the amorphous material in the preparation but was not seen in those areas of membrane bilayer free of amorphous material (Fig. $9 b$ ). Nonimmune serum or antibody that had been preadsorbed with pure GAP-43 did not bind to the membrane skeleton (data not shown).

Proteins that form the membrane skeleton of other cell types are also constituents of the membrane skeleton fraction prepared from IGCs

We investigated whether fodrin, vinculin, a-actinin, and talin are components of the membrane skeleton of IGCs by Western blotting 1-dimensional SDS gels and then incubating the blots with antibodies against these proteins. Fodrin, a-actinin, and talin immunoreactivities were all detectable in the membrane skeleton fraction (Fig. 10). In contrast, we were unable to detect anti-vinculin immunoreactivity in the membrane skeleton (Fig.

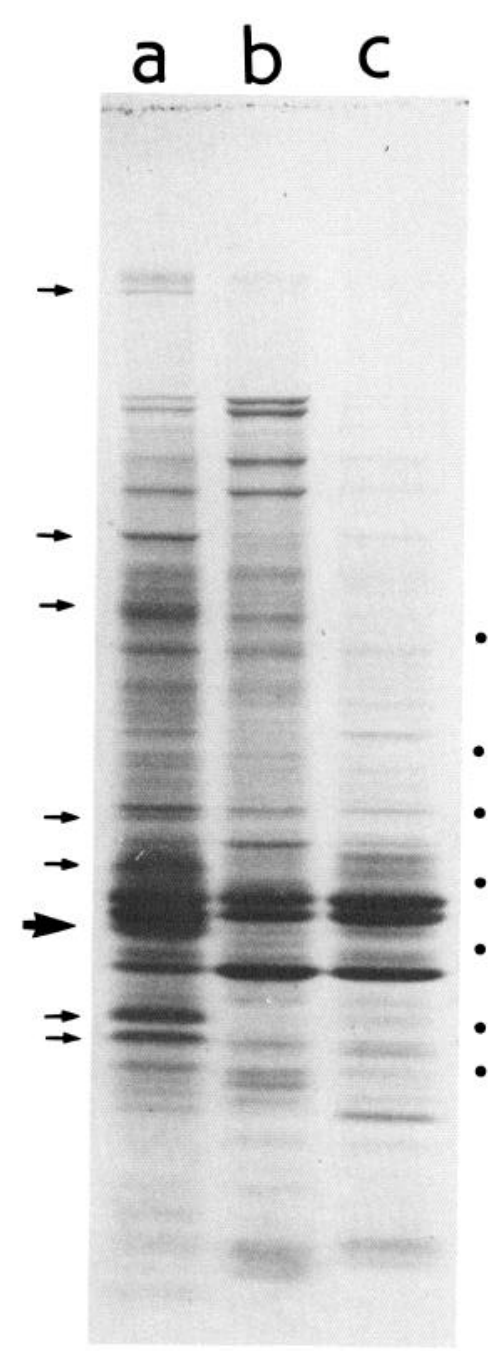

Figure 6. Certain proteins are specifically enriched in the IGC membrane skeleton. Coomassie-stained 3.5-15\% gradient SDS gel of proteins derived from the discontinuous sucrose gradient used to prepare membrane skeletons. $a$, The $10-30 \%$ interface, where the membrane skeleton is located. The specific proteins that are enriched in this fraction are indicated by small arrows and have molecular weights of 480,290, 220, $105,69,50,38$, and $33 \mathrm{kDa}$. Large arrow at $50 \mathrm{kDa}$ marks the position of GAP-43. $b$, Protein that pellets through the gradient. $c$, Whole growth cone particulate fraction for comparison. In each case $25 \mu \mathrm{g}$ of protein was loaded. Molecular weight standards are indicated on the right-hand side by dots; from top to bottom $180,116,97,58,48,36$, and $26 \mathrm{kDa}$.

10). This may be due to a weak antibody signal since the positive control (P1 rat forebrain membranes) was also poorly immunoreactive (results not shown). We tried 2 other commercially available anti-vinculin antibodies (from Sigma Chemical Co., St. Louis, MO, and from Brain Research Laboratories), but were unable to improve this result (data not shown). These results show that the membrane skeleton fraction prepared from IGCs contains proteins that have been identified in membrane skeletons of other cell types (Bennett, 1985).

\section{Discussion}

In order to determine GAP-43 localization in growth cones and characterize its interactions with other growth cone proteins, we have used as a model system a preparation of growth cones isolated from neonatal rat forebrains (Gordon-Weeks and Lock- 

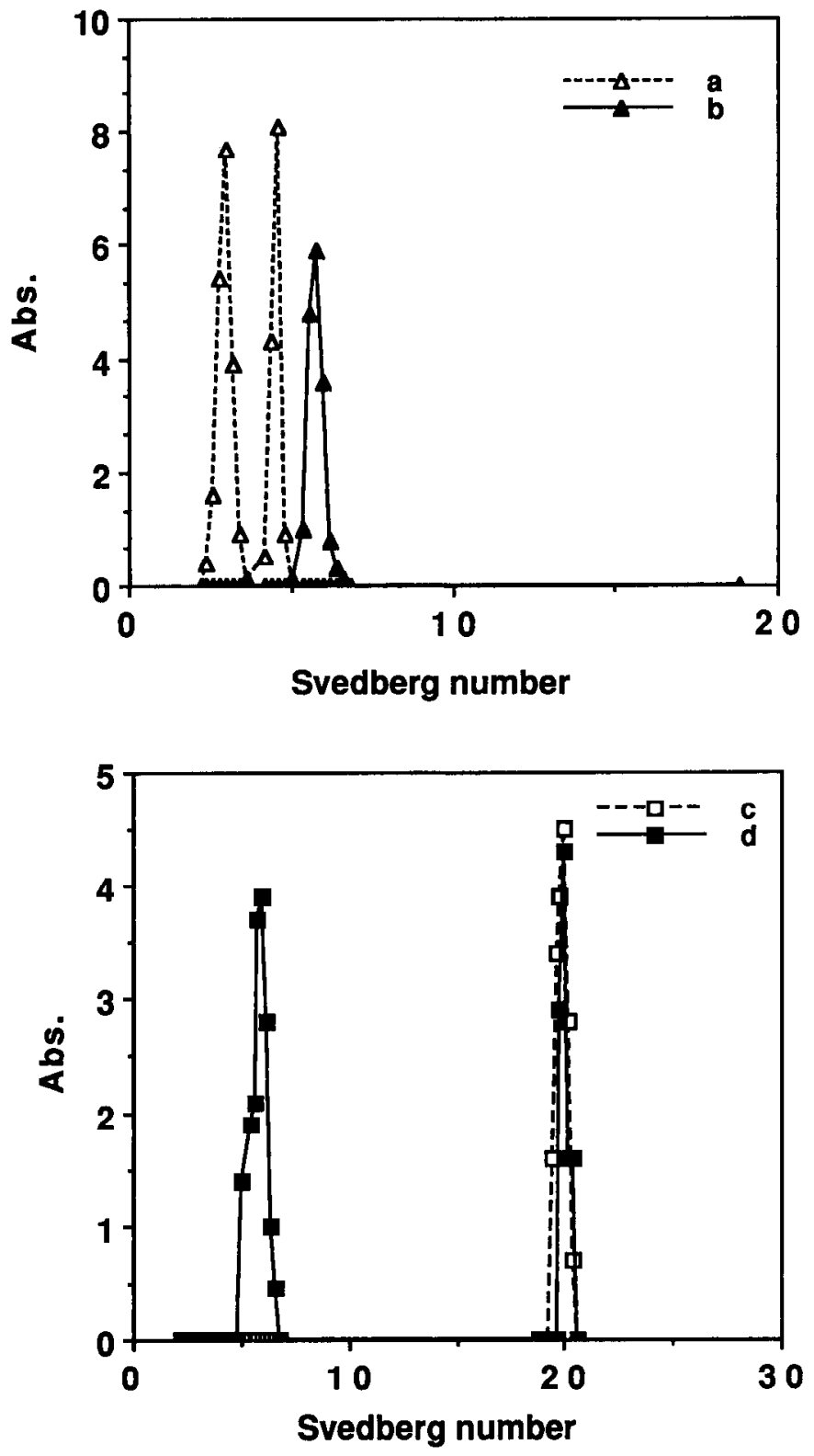

Figure 7. Association of GAP -43 with the membrane skeleton is not due to aggregation. Proteins were centrifuged on a 5-20\% continuous sucrose density gradient, and aliquots dot-blotted onto nitrocellulose paper. Protein location was visualized by naphthol blue-black staining or anti-GAP-43 immunoreactivity detected with biotin-avidin HRP. Densitometry was performed on transparent dot blots, and calculated Svedberg numbers plotted against absorbance in arbitrary units. $a(-\Delta-)$, Chymotrypsinogen and BSA (10 $\mu \mathrm{g}$ each) migrated at $2.7 \mathrm{~S}$ and $4.5 \mathrm{~S}$, respectively. $b(-\Delta-)$, Pure GAP-43 when centrifuged on a similar gradient migrated at $5.7 \mathrm{~S}$, equivalent to a molecular weight of $120 \mathrm{kDa}$. $c(-\square-)$, Membrane skeleton proteins $(30 \mu \mathrm{g})$ were centrifuged; antiGAP-43 immunoreactivity is detected at $20 \mathrm{~S}$; the apparent molecular weight of the membrane skeleton proteins. No immunoreactivity was detected elsewhere on the density gradient. $d(-\mathbf{-}-)$, Pure GAP-43 was centrifuged together with the membrane skeleton fraction; immunoreactivity was detectable at $6 \mathrm{~S}$ as well as $20 \mathrm{~S}$.

erbie, 1984). The morphology of growth cones isolated by this procedure is well preserved, and they retain the ability to take up gamma-aminobutyric acid and noradrenaline and subsequently to release them following depolarization with potassium (Gordon-Weeks et al., 1984; Lockerbie et al., 1985), as do growth

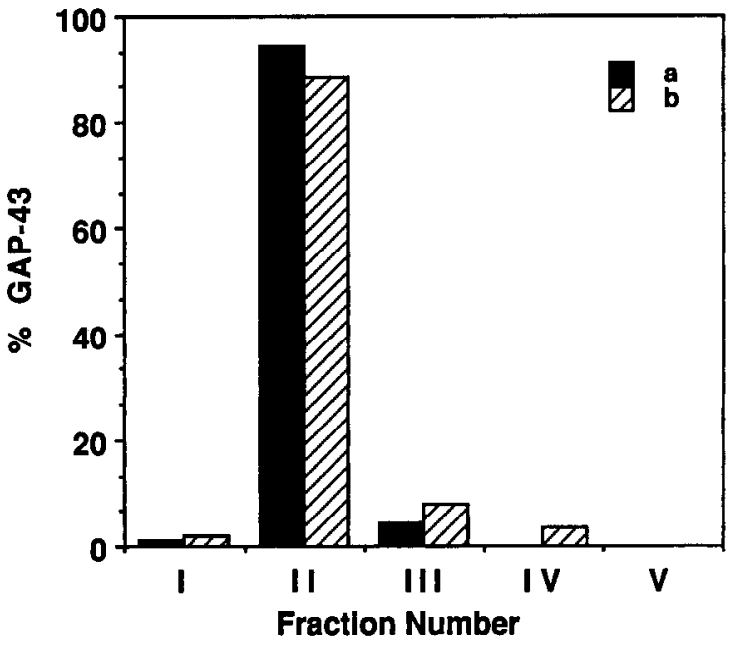

Figure 8. GAP-43 association with the membrane skeleton is not calcium dependent. The discontinuous sucrose gradient from the membrane skeleton preparation of IGCs that had been isolated in the absence of excess EGTA (dark bars), or in its presence (diagonals), was aliquoted into 5 6-ml zones, $I$, top; $I I, 10-30 \%$ interface; $I I I$, between interfaces; $I V, 30-50 \%$ interface; $V$, the bottom $6 \mathrm{ml}$ including the pellet. Twentymicroliter aliquots from each zone were separated on SDS gels and Western-blotted. Immunoreactivity was visualized with biotin-avidin HRP and densitometry at $415 \mathrm{~nm}$ performed on transparent blots. There is no significant difference in the localization of GAP-43 in the presence or absence of EGTA.

cones in tissue culture (Hume et al., 1983; Young and Poo, 1983), indicating that the preparation maintains the degree of physiological and structural integrity necessary for these studies.

GAP-43 immunoreactivity in IGCs that had settled onto a polyornithine substrate was intracellular, appeared punctate, and extended throughout the particles and their fine filopodia-like processes, as in the growth cones of neurites in tissue culture. From a total of 14 fields each containing 150 particles, $74.41 \%$ of the particles reacted with anti-GAP-43 antibody. We do not know the nature of the GAP-43 negative particles, they may be derived from dendritic growth cones that do not contain GAP43 (Goslin et al., 1988), or alternatively, they may be from parts of the neuron that are not strongly immunoreactive with this antibody, such as axons (Meiri et al., 1988), or they may be derived from nonneuronal cells (Gordon-Weeks and Lockerbie, 1984). Immunoreactivity in IGCs, as in cultured growth cones, was associated with areas of membrane that remained tightly attached to the substrate when the IGC was dislodged with a stream of buffer.

When IGCs were centrifuged at $100,000 \times g_{\mathrm{av}}$ for $75 \mathrm{~min}$ in a low-ionic-strength buffer, only $58 \%$ of the total IGC GAP-43 was pelleted. We have confined our observations to the "particulate" form of GAP-43 pelleted by this step, but the question remains as to the molecular nature of the GAP-43 remaining in the supernatant. We cannot assume that this GAP-43 is soluble since very small vesicle fragments (and, hence, maybe associated GAP-43) would not be pelleted by this step (Huttner et al., 1983). Nevertheless, fatty acid acylation of GAP-43 in growth cones is dynamic (Skene and Virag, 1989), implying that a soluble form of the protein may exist.

Treating this preparation of IGCs with Triton X-100 at a detergent : protein ratio of $166: 1$ (wt:wt) removed all but $10 \%$ of the particulate GAP-43 in IGCs. This type of extraction produces a cytoskeleton that under the electron microscope is 

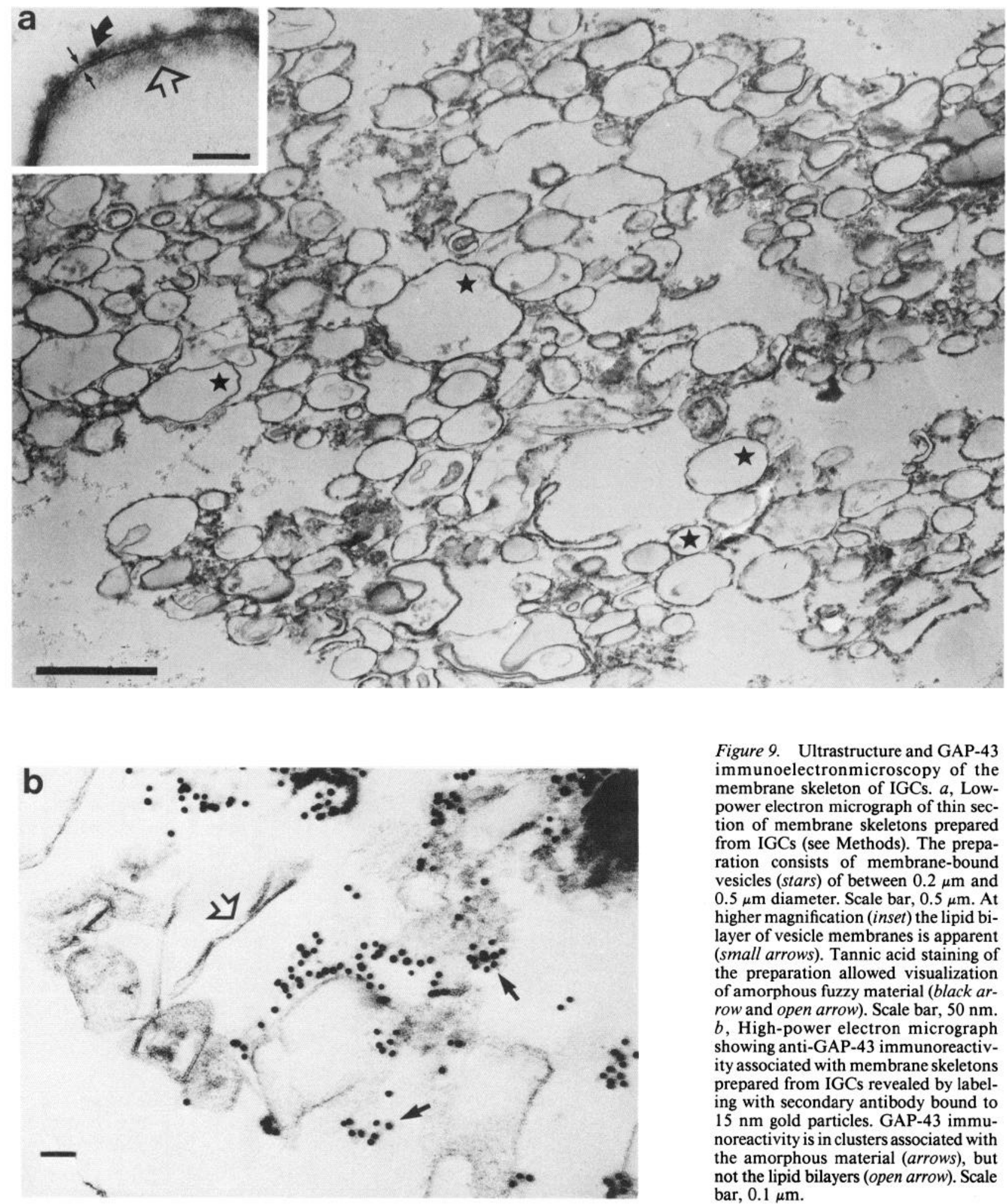

Figure 9. Ultrastructure and GAP-43 immunoelectronmicroscopy of the membrane skeleton of IGCs. $a$, Lowpower electron micrograph of thin section of membrane skeletons prepared from IGCs (see Methods). The preparation consists of membrane-bound vesicles (stars) of between $0.2 \mu \mathrm{m}$ and $0.5 \mu \mathrm{m}$ diameter. Scale bar, $0.5 \mu \mathrm{m}$. At higher magnification (inset) the lipid bilayer of vesicle membranes is apparent (small arrows). Tannic acid staining of the preparation allowed visualization of amorphous fuzzy material (black arrow and open arrow). Scale bar, $50 \mathrm{~nm}$. $b$, High-power electron micrograph showing anti-GAP-43 immunoreactivity associated with membrane skeletons prepared from IGCs revealed by labeling with secondary antibody bound to $15 \mathrm{~nm}$ gold particles. GAP-43 immunoreactivity is in clusters associated with the amorphous material (arrows), but not the lipid bilayers (open arrow). Scale bar, $0.1 \mu \mathrm{m}$

seen to consist largely of actin microfilaments (Gordon-Weeks, $1987 \mathrm{~b}$ ), so we interpret these results to mean that GAP-43 is not tightly bound to these nonextractable actin filaments.

In contrast, when IGCs were incubated with NP-40 at a lower detergent : protein ratio of 5:1 (wt:wt), and the insoluble proteins further fractionated by discontinuous sucrose density gradient centrifugation, $95-100 \%$ of the GAP-43 in IGCs was not solubilized and was located at the $10-30 \%$ interface on the gradient. This fraction has been characterized as a membrane skeleton because it contained glycoproteins as well as tubulin, actin, and actin-binding proteins (Moss, 1983). Furthermore, when this membrane skeleton was prepared from embryonic chicken fore- 


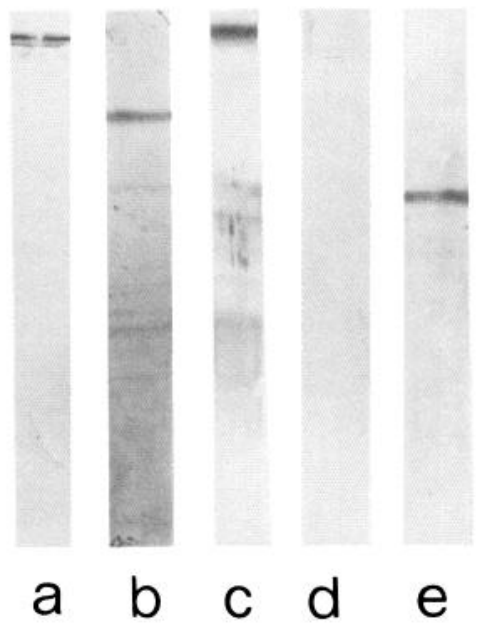

Figure 10. Identification of components of the IGC membrane skeleton. Western blots of proteins derived from the membrane skeleton fraction of IGCs that had been run on $10 \%$ SDS gels. Immunoreactivity was visualized with biotin-avidin HRP: $(a)$ anti-fodrin, $(b)$ anti-a-actinin, (c) anti-talin, (d) anti-vinculin, $(e)$ anti-GAP-43. In each case 30 $\mu \mathrm{g}$ of protein was loaded onto the gel.

brain, a major component was a protein that has been designated 3D5, which resembles GAP-43 in many respects (Allsop and Moss, 1989).

A similar approach of controlled detergent extraction has been described by Cheng and Sahyoun (1988) to prepare a cytoskeletal fraction from another preparation of IGCs (Pfenninger et al., 1983) by extracting them at a Triton X-100: protein ratio (7:1 wt:wt) lower than we used for our cytoskeleton preparation. When we repeated this experiment, almost all of the GAP-43 copurified with this cytoskeleton (results not shown). However, we believe that incubation of IGCs with this lower amount of nonionic detergent actually produces a combination of membrane skeleton and cytoskeleton, and that the subsequent discontinuous sucrose gradient centrifugation step that we use to prepare membrane skeletons, and which is not present in their method, then serves to separate these 2 structural components. This hypothesis is supported by the ultrastructural appearance of their cytoskeleton fraction under the electron microscope; it is a complex lattice of interconnected filaments which closely resembles the filamentous cytoskeleton of isolated growth cones (Gordon-Weeks, 1987b).

In growth cones from neurites in tissue culture and in IGCs, immunoelectron microscopy reveals GAP-43 immunoreactivity is located adjacent to the plasma membrane (van Lookeren Campagne et al., 1989), where the membrane skeleton is found. Electron microscopy of the membrane skeleton fraction we prepared showed that it is composed of lipid bilayers that in some areas are associated with amorphous fuzzy material clearly revealed by tannic acid staining. (One possible explanation for the presence of intact membrane in the fraction is that the low concentration of detergent present is insufficient to fully solubilize all the lipid and protein components of the fraction, thus leaving some membrane intact.) It is significant, however, that immunoelectron microscopy revealed that GAP-43 immunoreactivity is localized nonuniformly in clusters that were associated with the amorphous material but not with membrane bilayers that are devoid of this material, since it suggests that GAP-43 may not be inserted into the lipid bilayer, despite its fatty acid acylation. This result raises the question of the nature of the interaction that causes the association of GAP-43 with the membrane skeleton as well as with areas of the membrane that are tightly attached to the substrate.

Because we could not resolve the structure of the amorphous material, it was important to demonstrate that GAP-43 association with the membrane skeleton fraction was not due to artifactual entrapment or condensation of protein during the detergent lysis step, as had been described to occur when membrane skeletons are prepared from platelets (Fox, 1987). We, therefore, tried a variety of conditions to separate GAP-43 from the fraction: GAP-43 immunoreactivity remained with the membrane skeleton supramolecular aggregate during centrifugation through a continuous sucrose gradient and no additional GAP-43 became associated with the membrane skeleton when pure GAP-43 and membrane skeleton proteins were centrifuged together, demonstrating that the membrane skeleton does not nonspecifically adsorb GAP-43. In a second series of experiments we found that the presence of excess EGTA during all stages of IGC and subsequent membrane skeleton preparation did not affect the association of GAP-43 with the membrane skeleton. The latter experiment showed that, unlike protein kinase C, GAP-43 association with these membranes is not altered in the presence of calcium and is significant because it demonstrates that while GAP-43 copurifies with kinase C under certain circumstances (Aloyo et al., 1983), it does not similarly translocate from cytosol to membranes. Furthermore, these experiments show that if recruitment of GAP-43 from a soluble pool to the membrane skeleton does occur, it is not regulated solely by extracellular calcium.

In order to examine how GAP-43 interacts with proteins in the membrane skeleton fraction, we have begun to characterize its constituents. Actin and tubulin could be identified by their molecular weights and fodrin, a-actinin and talin were detected by immunoreactivity. Finally, a number of proteins that were specifically enriched in the membrane skeleton fraction were identified on SDS gels. We hope to use this information to address the question of which proteins of the membrane skeleton fraction are specifically associated with GAP-43; a central issue regarding the function of GAP-43 in the growth cone.

The immunohistochemical identification of GAP-43 in substrate-attached patches of membrane, and the exclusive localization of GAP-43 in the membrane skeleton of IGCs, suggest that this subcellular fraction also contains proteins from areas of focal contact. By analogy with fibroblasts where areas of focal contact in microspikes and ruffles can be differentiated (Rinnerthaler et al., 1988), it is likely that zones of focal contact in growth cones also perform diverse functions. At the moment we cannot tell whether GAP-43 is specifically enriched in these substrate-associated patches or whether they are derived from particular areas of the growth cone, such as filopodia or lamellipodia.

The membrane skeleton was originally described in erythrocytes as a structure that stabilizes the membrane and maintains cell shape, while allowing deformations to occur (Bennett, 1985), but the dynamic properties of the membrane skeleton are better illustrated in platelets, where shape changes during activation are more dramatic (Fox et al., 1988). The ability to change shape, and also direction, in response to external stimuli is also required of motile growth cones (Gundersen and Barrett, 1980; Carbonetto et al., 1983; Tomaselli et al., 1986), and because these functions are critical for successful axon outgrowth 
(Bentley and Toroian-Raymond, 1986), the association of GAP43 with the membrane skeleton is significant. Moreover, the location of GAP-43 in areas that are attached to the substrate means that it may be specifically implicated in substrate-growth cone interactions. This leads us to speculate that the failure of GAP-43 to be induced in injured mature mammalian CNS neurons (Skene and Willard, 1981a; Kalil and Skene, 1986) would thus make the growth cones unable to respond to specific stimuli even if the damaged axon retained the ability to elongate were it to be supplied with a permissive environment (Aguayo et al., 1987).

\section{References}

Aguayo, A. J., M. Vidal-Sanz, M. P. Villegas-Perez, and G. M. Bray (1987) Growth and connectivity of axotomized retinal neurons in adult rats with optic nerves substituted by PNS grafts linking the eye and the midbrain. Ann. N.Y. Acad.Sci. 495: 1-9.

Allsop, T. E., and D. J. Moss (1989) A developmentally regulated chicken neuronal protein associated with the cortical cytoskeleton. J. Neurosci. 9: 13-25.

Aloyo, V. J., H. Zwiers, and W. H. Gispen (1983) Phosphorylation B-50 protein by calcium-activated, phospholipid-dependent protein kinase and B-50 protein kinase. J. Neurochem. 41: 649-653.

Basi, G. S., I. V. Jacobson, J. Schilling, and J. H. P. Skene (1987) Primary structure and transcriptional regulation of GAP-43, a protein associated with nerve growth. Cell 49: 785-791.

Bennet, V. (1985) The membrane skeleton of human erythrocytes and its implications for mole comparisons. Annu. Rev. Biochem. 54:273304.

Benowitz, L. I., N. I. Perrone-Bizzozero, and S. P. Finklestein (1987) Molecular properties of the growth-associated protein GAP-43 (B50). J. Neurochem. 48: 1640-1647.

Bentley, D., and A. Toroian-Raymond (1986) Disoriented pathfinding by pioneer neurone growth cones deprived of filopodia by cytochalasin treatment. Nature 323: 712-715.

Bloch, R. J. (1984) Isolation of acetylcholine receptor clusters in substrate-attached material from cultured rat myotubes using saponin. J. Cell. Biol. 99: 984-993.

Bradford, M. M. (1976) A rapid and sensitive method for the quantification of microgram quantities of protein utilizing the principle of protein-dye binding. Anal. Biochem.72: 248-254.

Bray, D. (1982) Filopodial contraction and growth cone guidance. In Cell Behaviour, R. Bellairs, A. Curti, and G. Dunn, eds., pp. 299317, Cambridge U. P., Cambridge.

Carbonetto, S., M. M. Gruver, and D. C. Turner (1983) Nerve fiber growth in culture on fibronectin, collagen and glycosaminoglycan substrates. J. Neurosci. 3: 2324-2335.

Cheng, N., and N. Sahyoun (1988) The growth cone cytoskeleton. J. Biol. Chem. 263: 3935-3942.

Dosemeci, A., and R. Rodnight (1987) Demonstration by phase partitioning in Triton X-114 solutions that phosphoprotein B-50 (F1) from rat brain is an integral membrane protein. Neurosci. Lett. 74: 325-330.

Fox, J. E. B. (1987) The platelet cytoskeleton. In Thrombosis and Haemostasis, M. Verstraete, J. Vermylen, H. R. Lijnen, and J. Arnout, eds. pp. 175-225, International Society on Thrombosis and Haemostasis and Leuven U. P., Leuven, Belgium.

Fox, J. E. B., J. K. Boyles, M. C. Berndt, P. K. Steffan, and L. K. Anderson (1988) Identification of a membrane skeleton in platelets. J. Cell Biol. 106: 1525-1538.

Gispen, W. H., J. L. M. Leunissen, A. B. Oestereicher, A. J. Verkleij, and $\mathrm{H}$. Zwiers (1985) Presynaptic localization of B-50 phosphoprotein: The ACTH-sensitive protein kinase substrate involved in rat brain phosphoinositide metabolism. Brain Res. 328: 381-385.

Gogstad, G. O., and M. G. Krutnes (1982) Measurement of protein in cell suspensions using the Coomassie brilliant blue dye-binding assay. Anal. Biochem. 126: 355-359.

Gordon-Weeks, P. R. (1987a) Isolation of synaptosomes, growth cones and their subcellular components. In Neurochemistry: A Practical Approach, A. J. Turner and H. S. Bachelard, eds., pp. 1-26, IRL Press, Oxford.
Gordon-Weeks, P. R. (1987b) The cytoskeletons of isolated, neuronal growth cones. Neuroscience 21: 977-989.

Gordon-Weeks, P. R., and R. D. A. Lang (1988) The $\alpha$-tubulin of the growth cone is predominantly in the tyrosinated form. Dev. Brain Res. 470: 156-160.

Gordon-Weeks, P. R., and R. O. Lockerbie (1984) Isolation and partial characterization of neuronal growth cones from neonatal rat forebrain. Neuroscience 13: 119-136.

Gordon-Weeks, P. R., R. O. Lockerbie, and B. R. Pearce (1984) Uptake and release of $\left[{ }^{3} \mathrm{H}\right] \mathrm{GABA}$ by growth cones isolated from neonatal rat brain. Neurosci. Lett. 52: 205-210.

Goslin, K., D. J. Schreyer, J. H. P. Skene, and G. Banker (1988) Development of neuronal polarity: GAP-43 distinguishes axonal from dendritic growth cones. Nature 336: 672-674.

Gundersen, R. W., and Barrett, J. N. (1980) Characterisation of the turning response of dorsal root neurites towards nerve growth factor. J. Cell Biol. 87: 546-554.

Hume, R. I., L. W. Role, and G. D. Fishbach (1983) Acetylcholine release from growth cones detected with patches of acetylcholine receptor-rich membranes. Nature 305: 632-634.

Huttner, W. B., W. Schiebler, P. Greengard, and P. De Camilli (1983) Synapsin I (protein 1), a nerve terminal-specific phosphoprotein. III. Its association with synaptic vesicles studied in a highly purified synaptic vesicle preparation. J. Cell Biol. 96: 1374-1388.

Jacobson, R. D., I. D. Virag, and J. H. P. Skene (1986) A protein associated with axon growth, GAP-43, is widely distributed and developmentally regulated in rat CNS. J. Neurosci. 6: 1843-1855.

Johnson, M. I., N. Kleitman, M. Willard, and K. F. Meiri (1987) GAP. 43 immunoreactivity is correlated with rate of growth cone translocation and with initiation of neurite outgrowth in cultured sympathetic neurons. Neurosci. Abstr. 413: 11.

Kalil, K., and J. H. P. Skene (1986) Elevated synthesis of an axonally transported protein correlates with axonal outgrowth in normal and injured pyramidal tracts. J. Neurosci. 6: 2563-2570.

Karns, L. R., S.-C. Ng, J. A. Freeman, and M. C. Fishman (1987) Cloning of complementary DNA for GAP-43, a neuronal growth related protein. Science 236: 597-600.

Laemmli, U. K. (1970) Cleavage of structural proteins during the preassembly of the head of bacteriophage T4. Nature 227: 680-685.

Letourneau, P. C. (1982) Nerve fibre growth and its regulation by extrinsic factors. In Neuronal Development, N. C. Spitzer, ed., pp. 213-254, Plenum, New York.

Lockerbie, R. O., P. R. Gordon-Weeks, and B. R. Pearce (1985) Growth cones isolated from developing rat forebrain: Uptake and release of GABA and noradrenaline. Dev. Brain Res. 21: 265-275.

Martin, R. G., and B. N. Ames (1961) A method of determining the sedimentation behavior of enzymes: application to protein mixtures. J. Biol. Chem. 236: 1372-1379.

Masure, H. R., K. A. Alexander, B. T. Wakim, and D. R. Storm (1986) Physicochemical and hydrodynamic characterization of P-57, a neurospecific calmodulin binding protein. Biochemistry 25: 7553-7560.

McIntosh, H., D. Parkinson, K. Meiri, N. Daw, and M. Willard (1989) A GAP-43-like protein in cat visual cortex. Visual Neuroscience 2: 583-591.

Meiri, K. F., and P. R. Gordon-Weeks (1987) Distribution of GAP43 within isolated neuronal growth cones. Neurosci. Abstr. 413: 16.

Meiri, K. F., K. H. Pfenninger, and M. Willard (1986) Growth associated protein, GAP-43, a polypeptide that is induced when neurons extend axons, is a component of growth cones and corresponds to pp46, a major polypeptide of a subcellular fraction that is enriched in growth cones. Proc. Natl. Acad. Sci. USA. 83: 3537-3541.

Meiri, K. F., M. Willard, and M. I. Johnson (1988) Distribution and phosphorylation of the growth associated protein GAP-43 in regenerating sympathetic neurons in culture. J. Neurosci. 8: 3537-3542.

Moss, D. J. (1983) Cytoskeleton-associated glycoproteins from chicken sympathetic neurons and chicken embryo brain. Eur. J. Biochem. 135: 291-297.

Oakley, B. R., D. R. Kirsch, and N. R. Morris (1980) A simplified ultrasensitive silver stain for detecting proteins in polyacrylamide gels. Anal. Biochem. 105: 361-363.

Pfenninger, K. H., L. Ellis, M. P. Johnson, L. B. Friedman, and S. Somlo (1983) Ncrve growth cones isolated from fetal rat brain. Subcellular fractionation and characterization. Cell 35: 573-584.

Read, S. M., and D. H. Northcote (1981) Minimization of variation 
in the response to different proteins of the Coomassie blue $\mathrm{G}$ dyebinding assay for protein. Anal. Biochem. 116: 53-64.

Rinnerthaler, G., B. Geiger, and J. V. Small (1988) Contact formation during fibroblast locomotion: Involvement of membrane ruffles and microtubules. J. Cell Biol. 106: 747-760.

Skene, J. H. P., and I. Virag (1989) Posttranslational membrane attachment and dynamic fatty acylation of a neuronal growth cone protein, GAP-43. J. Cell Biol. 108: 613-624.

Skene, J. H. P., and M. Willard (1981a) Axonally transported proteins associated with growth in rabbit central and peripheral nervous system. J. Cell. Biol. 89: 96-103.

Skene, J. H. P., and M. Willard (1981b) Electrophoretic analysis of axonally transported proteins in toad retinal ganglion cells. J. Neurochem. 37: 79-87.

Skene, J. H. P., R. D. Jacobson, G. J. Snipes, C. B. MacGuire, J. Norden, and J. A. Freeman (1986) A protein induced during nerve growth, GAP-43, is a major component of growth cone membranes. Science 233: 783-785.
Tomaselli, K. J., L. F. Reichardt, and J. L. Bixby (1986) Distinct molecular interactions mediate neuronal process outgrowth on nonneuronal cell surfaces and extracellular matrices. J. Cell Biol. 103: 2659-2672.

van Lookeren Campagne, M., A. B. Oestreicher, P. M. P. van Bergen en Henegouwen, and W. H. Gispen (1989) Ultrastructural immunocytochemical localization of B-50/GAP-43, a protein kinase $C$ substrate, in isolated presynaptic nerve terminals and nerve growth cones. J. Neurocytol. 18: 479-489.

Willard, M., K. F. Meiri, and M. I. Johnson (1987) The role of GAP43 in a axon growth. In Axonal Transport. Neurology and Neurobiology, Vol. 25, R. S. Smith and M. S. Bisby, eds., pp. 407-421, Liss, New York.

Young, S. H., and M.-M. Poo (1983) Spontaneous release of transmitter from growth cones of embryonic neurones. Nature 305: 634637. 